\title{
The human oral cavity microbiota composition during acute tonsillitis: a cross-sectional survey
}

Yun Kit Yeoh ${ }^{1,2,3}$, Man Hin Chan ${ }^{4}$, Zigui Chen ${ }^{1,2,3}$, Eddy W. H. Lam ${ }^{4}$, Po Yee Wong ${ }^{1,2}$, Chi Man Ngai ${ }^{4}$, Paul K. S. Chan ${ }^{1,2,3}$ and Mamie Hui ${ }^{1,2,3^{*}}$

\begin{abstract}
Background: Microbial culture-based investigations of inflamed tonsil tissues have previously indicated enrichment of several microorganisms such as Streptococcus, Staphylococcus and Prevotella. These taxa were also largely reflected in DNA sequencing studies performed using tissue material. In comparison, less is known about the response of the overall oral cavity microbiota to acute tonsillitis despite their role in human health and evidence showing that their compositions are correlated with diseases such as oral cancers. In addition, the influence of subject-specific circumstances including consumption of prescription antibiotics and smoking habits on the microbiology of acute tonsillitis is unknown.
\end{abstract}

Methods: We collected oral rinse samples from 43 individuals admitted into hospital for acute tonsillitis and 165 non-disease volunteers recruited from the public, and compared their microbial community compositions using $16 \mathrm{~S}$ rRNA gene sequencing. We assessed the impact of tonsillitis, whether subjects were prescribed antibiotics, the presence of oral abscesses and their smoking habits on community composition, and identified specific microbial taxa associated with tonsillitis and smoking.

Results: Oral rinse community composition was primarily associated with disease state (tonsillitis vs non-tonsillitis) although its effect was subtle, followed by smoking habit. Multiple Prevotella taxa were enriched in tonsillitis subjects compared to the non-tonsillitis cohort, whereas the non-tonsillitis cohort primarily showed associations with several Neisseria sequence variants. The presence of oral abscesses did not significantly influence community composition. Antibiotics were prescribed to a subset of individuals in the tonsillitis cohort but we did not observe differences in community composition associated with antibiotics consumption. In both tonsillitis and non-tonsillitis cohorts, smoking habit was associated with enrichment of several Fusobacterium variants.

Conclusions: These findings show that the oral cavity microbial community is altered during acute tonsillitis, with a consistent enrichment of Prevotella during tonsillitis raising the possibility of targeted interventions. It also supports the possible link between smoking, Fusobacteria and oral cancers.

Keywords: Mouth rinse, 165 ribosomal RNA gene, Microbial community, Prevotella, Smoking, Fusobacteria

\footnotetext{
*Correspondence: mamiehui@cuhk.edu.hk

${ }^{1}$ Centre for Gut Microbiota Research, Faculty of Medicine, The Chinese

University of Hong Kong, Hong Kong SAR, China

${ }^{2}$ Department of Microbiology, Faculty of Medicine, The Chinese University of

Hong Kong, Hong Kong SAR, China

Full list of author information is available at the end of the article
}

(c) The Author(s). 2019 Open Access This article is distributed under the terms of the Creative Commons Attribution 4.0 International License (http://creativecommons.org/licenses/by/4.0/), which permits unrestricted use, distribution, and reproduction in any medium, provided you give appropriate credit to the original author(s) and the source, provide a link to the Creative Commons license, and indicate if changes were made. The Creative Commons Public Domain Dedication waiver (http://creativecommons.org/publicdomain/zero/1.0/) applies to the data made available in this article, unless otherwise stated. 


\section{Background}

The human oral microbiome has been relatively well characterized [1-4], and ongoing efforts are focused on studying compositional and spatial organization of oral communities as well as similarities and differences across populations [5]. Another widely appreciated aspect is the role of oral microbial communities in oral and systemic diseases [6,7]. One common conditionthe inflammation of tonsils (termed tonsillitis), is also regarded to have a microbial aetiology. Tonsils are part of the lymphatic system and can become inflamed when infected by bacteria or viruses. While tonsillitis is usually a self-limiting disease, abscesses can form in some cases and require surgical intervention to drain accumulated pus. Culture-based characterization of microorganisms in tonsil tissues of patients with tonsillitis have reported a polymicrobial association commonly involving group A streptococci, Staphylococcus aureus, Streptococcus pneumoniae, Haemophilus influenzae, and members of the Prevotella, Bacteroides, Fusobacterium, Porphyromonas and Veillonella genera [8-10]. Since many humanassociated microorganisms are not cultivable under laboratory conditions, recent studies have applied culture-independent DNA sequencing methods to identify the kinds of microorganisms present and quantify their abundances in the human microbiome [11]. By sequencing the microbial small subunit ribosomal RNA gene (16S), a study of microorganisms enriched in tonsillar tissue from adult subjects with recurrent tonsillitis identified enrichment of members of the Treponema, Fusobacterium, Streptococcus, Selenomonas, Gemella, Tannerella and Prevotella genera compared to healthy individuals [12].

While it has been reported that tonsil tissueassociated microbial communities are altered in subjects with tonsillitis, there are no studies describing whether the total oral cavity microbiome is also influenced by this condition. The oral cavity microbiome is commonly studied by collecting oral rinse samples $[11,13,14]$, and its composition has been shown to correlate with diseases such as oral cavity and oropharyngeal cancers [13, 15]. Here, we collected oral rinse samples from patients showing symptoms of acute tonsillitis and compared their oral cavity microbial community composition to healthy individuals without oral disease. We hypothesized that the oral microbiome composition in tonsillitis patients differed from healthy individuals, and that disease state (i.e. tonsillitis vs no disease) was the primary factor attributable to differences in community composition. We also investigated the influence of prescription antibiotics, the presence of oral abscesses and smoking habits on oral rinse community compositions.

\section{Methods}

\section{Subjects and sample collection}

Individuals admitted to the Department of Otorhinolaryngology, Head and Neck Surgery, Yan Chai Hospital, Hong Kong SAR for symptoms of acute tonsillitis were recruited for this observational study. No interventions were made based on the needs or findings of this study. Firstly, demographic (such as age, gender, smoking habits) and medical information (such as antibiotics prescribed prior to admission) were collected. Disease severity was noted based on whether subjects required incision and drainage of abscesses. Before any treatment or medication was prescribed, subjects first rinsed their mouths with drinking water to remove any food debris. They then swilled $10 \mathrm{ml}$ of sterile $0.9 \%$ saline solution in their oral cavities for $1 \mathrm{~min}$, and collected the saline rinse in sterile $70 \mathrm{ml}$ specimen jars. A separate cohort of healthy volunteers was recruited from the public to provide oral rinse samples using the same collection method to serve as a reference for the oral microbiome in the local population. Demographic information was recorded through a self-administered validated questionnaire (Additional file 2). All oral rinse samples were stored at $-20^{\circ} \mathrm{C}$ until DNA extraction.

\section{DNA extraction and $16 \mathrm{~S}$ amplicon sequencing}

DNA extraction and $16 \mathrm{~S}$ amplicon sequencing were performed as previously described [16]. Briefly, DNA was extracted from $1 \mathrm{ml}$ of oral rinse using the QIAGEN DNeasy PowerSoil Kit according to manufacturer's instructions, and the V3 and V4 variable regions of the $16 \mathrm{~S}$ gene were amplified by polymerase chain reaction (PCR). DNA-free negative and mock microbial community positive controls (ZymoBIOMICS Microbial Community DNA Standard, catalogue number D6305) were included in the PCR. Following amplification, sequencing adapters and multiplex indices were added to the PCR products in a second PCR and the resulting amplicons purified using the QIAquick Gel Extraction Kit. Purified final products were sequenced on an Illumina MiSeq $(2 \times 300$ bp) using the v3 MiSeq Reagent Kit.

\section{$16 \mathrm{~S}$ sequence data processing}

Demultiplexed raw sequence data were imported into QIIME 2 v2018.6 [17]. Using the DADA2 workflow in QIIME 2, primer and low quality sequences were trimmed, and remaining reads subsequently denoised and merged. Alpha diversity metrics (species richness, Shannon index and Faith's phylogenetic diversity) were calculated based on sequence counts normalised to a depth of 4000 sequences per sample. To assign taxonomy to sequences, a classifier was first trained on reference $16 \mathrm{~S}$ sequences extracted from the SILVA $16 \mathrm{~S}$ database release 128 [18] using the $16 \mathrm{~S}$ gene V3-4 
universal primer sequences. This classifier was then run on representative sequences produced by DADA2 to assign probable taxonomies to the corresponding sequences. The final non-normalized counts table based on exact sequence variants (ESVs) and their $16 \mathrm{~S}$ taxonomies, as well as UniFrac distances of community composition were exported from QIIME 2, and used as input in $\mathrm{R}$ for statistical analysis.

\section{$16 \mathrm{~S}$ amplicon-based microbial community composition analyses}

The resulting ESV counts table from QIIME 2 was imported together with sample metadata into R v3.5.1. A centered log-ratio transformation was applied to ESV counts before downstream analyses to ensure that the counts fulfilled assumptions of independence between predictor variables for statistical analyses (explained in [19]). Permutational multivariate analysis of variance (PERMANOVA) was used to assess whether factors such as disease state (tonsillitis vs healthy), smoking habit, age and gender significantly influenced community composition, as well as to determine the amount of variation in community composition attributable to each of these factors. Principal component analysis (PCA) and principal coordinate analysis (PCoA) ordinations were used to visualise the clustering of samples based on their compositional similarities. Association of ESVs to experimental factors were identified using the linear discriminant analysis effect size (LEfSe) algorithm [20] implemented in the Huttenhower Lab Galaxy web application framework available online (http://huttenhower. sph.harvard.edu/galaxy/), and also with a random forest classifier. PERMANOVA, PCA and PCoA are implemented in the vegan $R$ package v2.4-6, power calculations in the HMP $\mathrm{R}$ package [21], and random forest in the randomForest $\mathrm{R}$ package $\mathrm{v} 4.6-14$. Figures were edited in Inkscape v0.92 for clarity.

\section{Results}

Oral rinse microbiome composition of healthy individuals and subjects with tonsillitis

A total of 43 subjects with acute tonsillitis were recruited to assess the oral rinse microbial community during tonsillitis. Of these subjects, 15 were prescribed antibiotics prior to admission, and 20 required incision and drainage of abscesses (Table 1). Their mean white cell counts measured as per hospital admissions procedure was $15.1 \times 10^{9}$ cells $/ \mu$ l blood (median $13.9 \times 10^{9}$ cells $/ \mu \mathrm{l}$ ), of which $76.9 \%$ on average was comprised of neutrophils suggesting a bacterial aetiology. As a reference non-tonsillitis cohort, 165 healthy volunteers were recruited from the public. These healthy subjects reported no history of chronic diseases or chronic use of medication and antibiotics in the past year. Sequencing of PCR amplicons from these 208 oral rinse samples produced $3,162,740$ sequences, with a median of 14,909 sequences per sample after quality filtering and merging read pairs. With this design and sequencing depth, the study had $85.3 \%$ power to detect differences in community composition between tonsillitis and healthy subjects ( significance level $=0.05$, number of reads per sample $=$ 14,000).

To determine whether oral microbial community composition significantly differed between tonsillitis patients and healthy subjects, we applied a centered log-ratio transformation on $16 \mathrm{~S}$ counts data and then performed a PCA. Similarly, we also performed a PCoA on weighted UniFrac distances that account for relatedness among members of the community. In both ordinations, samples primarily clustered according to disease state (i.e. tonsillitis vs healthy cohorts) (Fig. 1) $(p<0.001$, permutation test), indicating that the oral rinse microbiome composition was significantly altered in patients with tonsillitis relative to healthy individuals. These observations were supported by a PERMANOVA (stratified by cohort) indicating that cohort (i.e. tonsillitis vs healthy), smoking habit (current, former and non-smokers), age and gender were significantly associated with microbial community composition in descending size of effect (Table 2). Alpha diversity, however, did not differ between the tonsillitis and healthy cohorts (Additional file 1: Figure S1) ( $p>0.05$, Kruskal-Wallis test).

\section{Microbial taxa associated with healthy individuals and subjects with tonsillitis}

As there was no interaction between tonsillitis disease status with smoking habit, age or gender indicated by PERMANOVA, we directly compared community composition between the tonsillitis and non-tonsillitis cohorts to identify differentially enriched taxa. Using the

Table 1 Characteristics of the study subjects

\begin{tabular}{lll}
\hline Characteristic & Healthy cohort $(N=165)$ & Tonsillitis cohort $(N=43)$ \\
\hline Male: female ratio & 2.2 & 2.3 \\
Median age (interquartile range) & $40(31)$ years & $36(23.5)$ years \\
Antibiotics (prior to sampling) & none & In 15 subjects \\
Abscesses (requiring incision and drainage) & none & In 20 subjects \\
Smokers & 17 former, 11 current, 137 non & 2 former, 18 current, 23 non \\
\hline
\end{tabular}




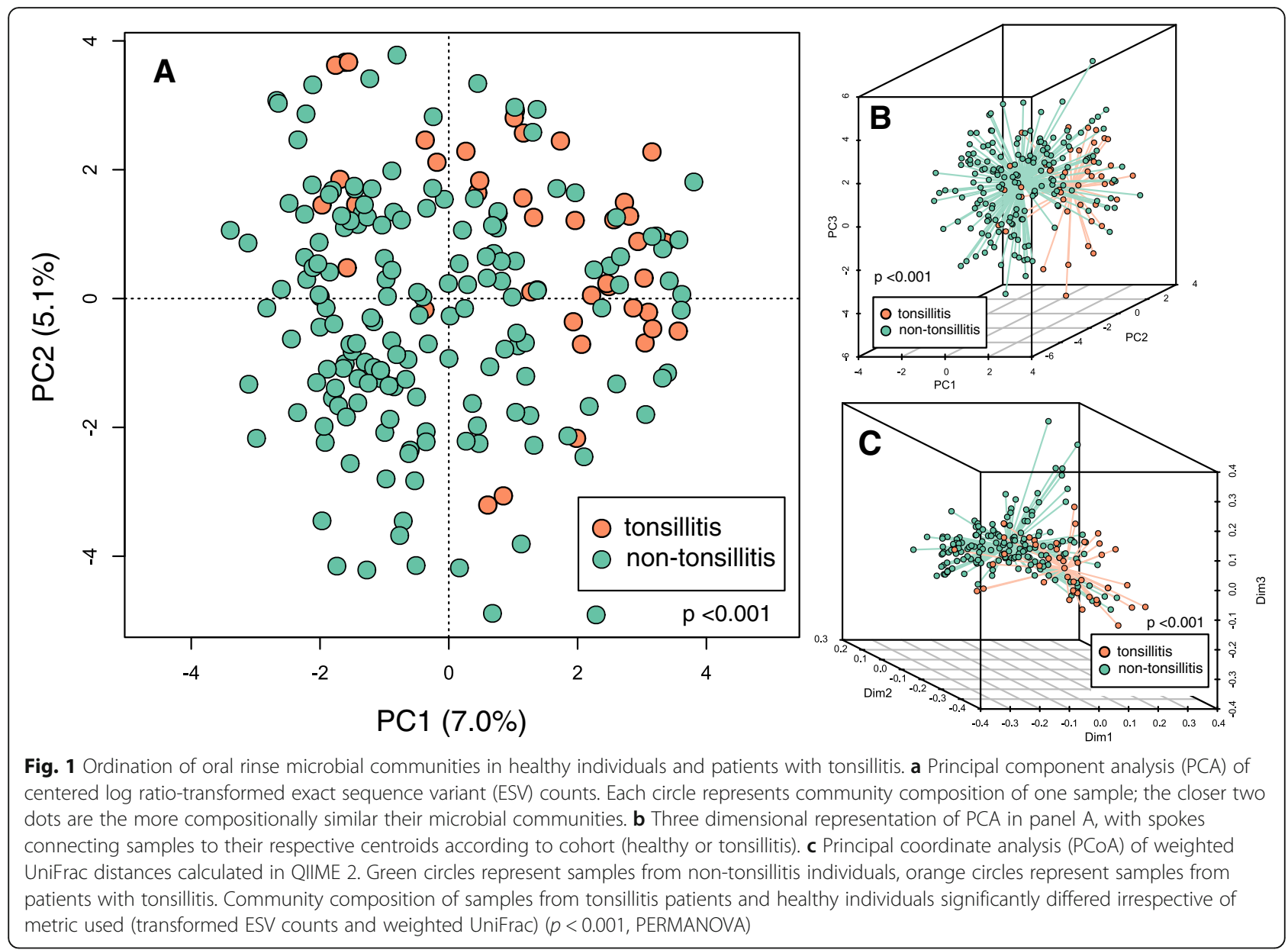

linear discriminant analysis (LDA) effect size (LEfSe) algorithm, 103 ESVs were significantly associated with the tonsillitis cohort whereas 57 were significantly associated with the healthy cohort $(p<0.05$, LDA score $>2)$. Five of the top 10 ESVs associated with the tonsillitis cohort were classified as Prevotella, and the remaining were a Streptococcus, two Veillonella, a Lactobacillus and an Atopobium (Fig. 2). In contrast, the oral rinse community of the healthy cohort was associated with Neisseria, Haemophilus, Fusobacterium, Streptococcus, Lautropia and a Rothia. These findings were consistent with those generated using a random forest classifier to compare community composition between tonsillitis subjects and healthy individuals, in which Atopobium, Veillonella and Prevotella were implicated in tonsillitis samples (Additional file 1: Figure S2).

\section{Secondary factors associated with oral microbiota composition in subjects with tonsillitis}

Prior to recruitment into this study, 15 subjects from the tonsillitis cohort were prescribed antibiotics by general practitioners for an average duration of 3.1 days (standard deviation of 2.5 days). Amoxicillin/clavulanate was prescribed to nine subjects, and amoxicillin, azithromycin, cefuroxime and levofloxacin to one subject each. Two subjects were uncertain of their prescribed antibiotics. In addition, 20 subjects underwent incision and

Table 2 PERMANOVA of oral microbial community composition in healthy and tonsillitis cohorts

\begin{tabular}{llllll}
\hline & Sums of squares & Mean squares & F model & $R^{2}$ & $p$-value \\
\hline Disease state & 8485 & 8484.6 & 4.032 & 0.019 & $<0.001$ \\
Smoking & 7360 & 3680.0 & 1.749 & 0.016 & $<0.001$ \\
Age & 3743 & 3743.4 & 1.779 & 0.008 & $<0.001$ \\
Gender & 3015 & 3014.8 & 1.433 & 0.007 & 0.0110 \\
Antibiotics & 2236 & 2236.4 & 1.063 & 0.005 & 0.3263 \\
Residuals & 423,001 & 2104.5 & & 0.945 & \\
\hline
\end{tabular}




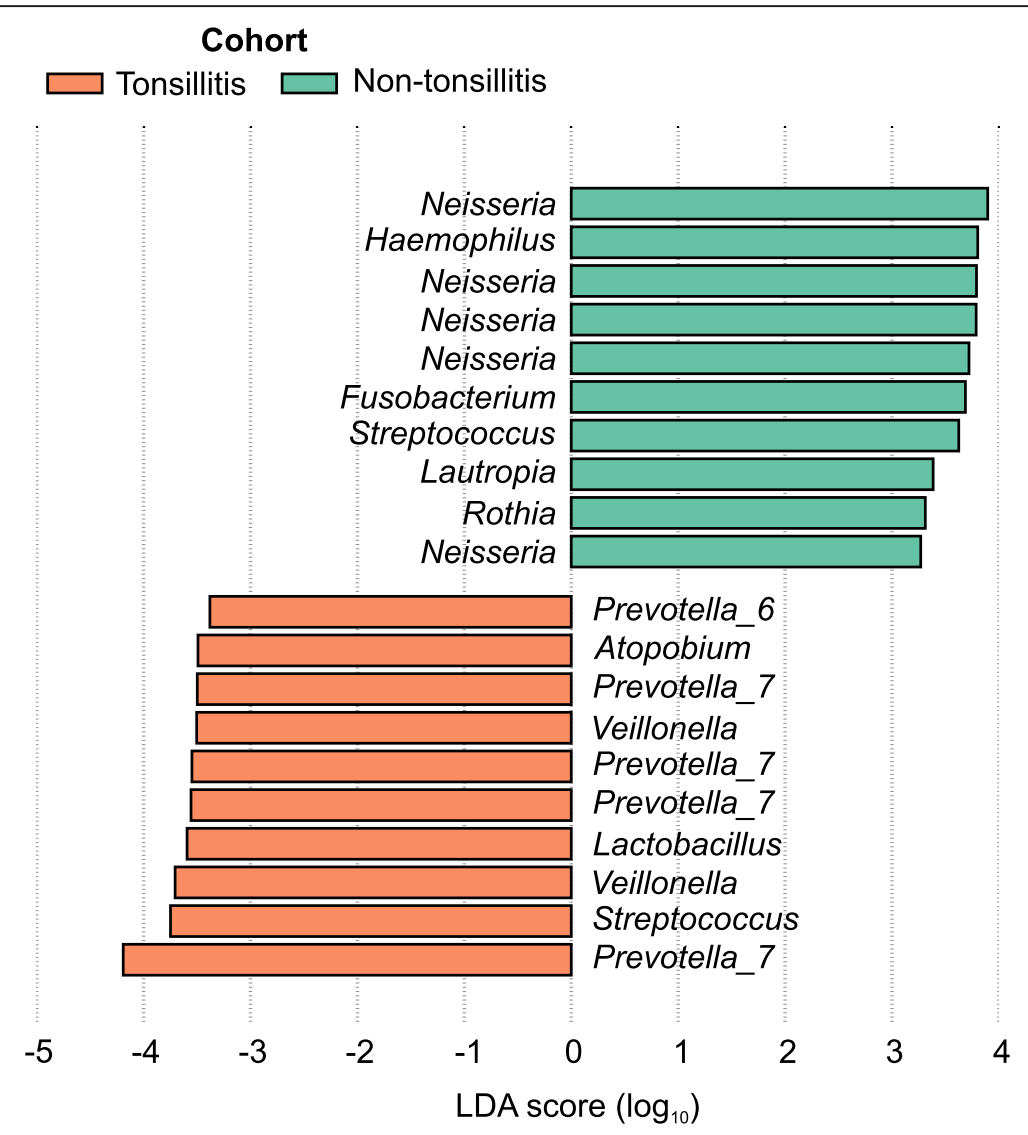

Fig. 2 Top 10 exact sequence variants (ESVs) associated with the tonsillitis and healthy cohorts ranked according to linear discriminant analysis (LDA) effect size. ESVs were identified using the LDA effect size (LEFSe) algorithm with default parameters. Taxonomy labels indicate genus level classification (or lowest rank available) with reference to the SILVA database. Green bars represent ESVs associated with non-tonsillitis individuals, orange bars represent ESVs associated with patients with tonsillitis

drainage of abscesses, suggesting that they experienced a more severe bout of disease. Since the consumption of antibiotics and disease severity are known to influence microbial community composition, we analysed data from the tonsillitis cohort alone to assess whether prescription of antibiotics and presence of abscesses requiring surgical intervention were associated with oral rinse community composition. A PERMANOVA indicated that community composition was not associated with antibiotics consumption, the presence of abscesses, age or gender, but identified a significant difference between smokers and non-smokers (Additional file 1: Table S1). These differences were attributed to associations of ESVs such as Rothia, Fusobacterium and Actinomyces with smokers, and multiple sequence variants of Neisseria, Veillonella, Prevotella and Granulicatella with nonsmokers although ESVs classified under the same genera were also detected in smokers (Fig. 3) (LEfSe; two former smokers were excluded). Differences in oral community composition were also detected in the healthy cohort in which smoking status, age and gender were significantly associated in descending size of effect
(Additional file 1: Table S2). Three fusobacterial ESVs were implicated in the oral rinse microbial communities in smokers and former-smokers from the healthy cohort, although another two were associated with non-smokers (Additional file 1: Figure S3). A Neisseria ESV was again the most strongly associated taxa in non-smokers. These results suggest that members of the Fusobacterium genus are enriched in the oral cavities of smokers, however, further validation is required to identify these taxa beyond the genus level resolution provided by conventional 16S rRNA gene sequencing.

\section{Discussion}

Tonsillitis is traditionally associated with an overgrowth of bacterial taxa such as Bacteroides, Fusobacterium, Veillonella, Prevotella, Streptococcus, Staphylococcus and Haemophilus based on laboratory cultures of tonsillar tissue and material from abscesses [8,22]. In addition to supporting these observations, culture-independent microbial community surveys based on DNA sequencing have also uncovered additional associations with other taxa such as Treponema, Fusobacterium, Gemella and 


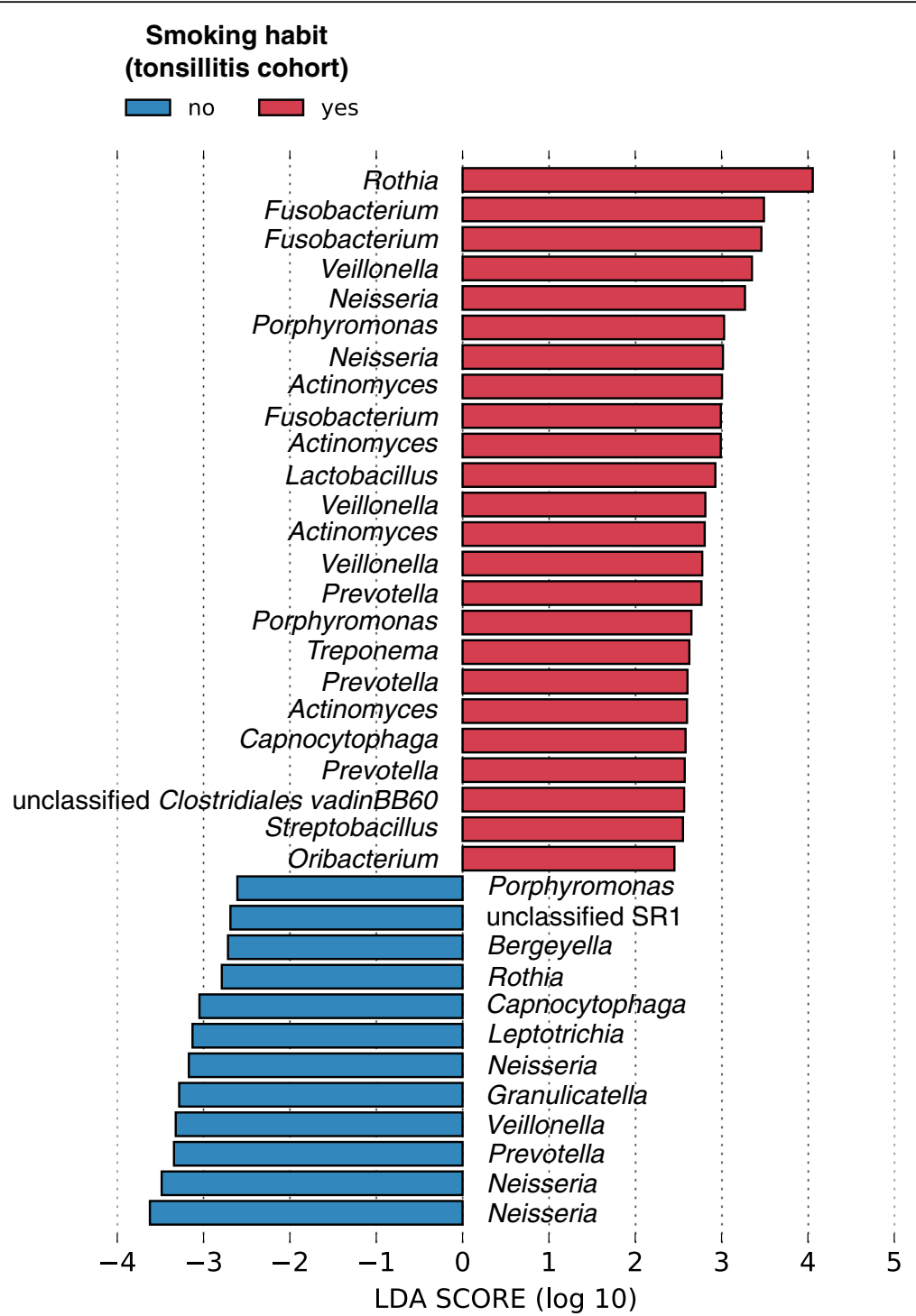

Fig. 3 Exact sequence variants (ESVs) associated with smokers and non-smokers in the tonsillitis cohorts ranked according to linear discriminant analysis (LDA) effect size. ESVs were identified using the linear discriminant analysis effect size (LEfSe) algorithm. Only ESVs with an LDA score of at least 2 in their respective groups are shown. Genus level classification (or lowest rank available) with reference to the SILVA database is provided. Red bars represent ESVs associated with smokers, whereas blue bars represent ESVs associated with non-smokers

Tannerella [12]. Our data extended these findings to a cohort of tonsillitis patients in Hong Kong in which we observed a subtle but significant difference in oral rinse microbial community composition compared to healthy individuals, in line with previous reports that composition of microbial communities in oral rinse samples is predictive of oral disease [13]. An even smaller effect of gender and age was identified, although these two factors were only significantly associated with oral community composition in healthy subjects when the tonsillitis and healthy cohorts were analysed separately. We postulate that the lack of association in tonsillitis subjects could be due to a larger influence of tonsillitis overriding effects linked to age and/or gender, and also partly because of reduced power in detecting age/gender differences in community composition in the smaller tonsillitis cohort compared to healthy individuals (Table 1).

Although compositional differences in oral community composition linked to tonsillitis were subtle as indicated by PERMANOVA and the lack of statistical difference in alpha diversity indexes between cohorts, five of the top 10 ESVs associated with tonsillitis consistently classified as Prevotella suggested that multiple members of this genus may be involved with oral disease. Indeed, Prevotella sequence variants have been implicated in various oral conditions such as periodontitis [23-25] and endodontic abscesses [26], and are possibly enriched through their preferential utilization of proteins and 
production of cytotoxic end products [27, 28]. We also identified several Streptococcus, Veillonella, Lactobacillus and Atopobium variants associated with the tonsillitis cohort. While these tonsillitis-associated taxa were mostly distinct from those associated with healthy samples, Streptococcus ESVs were linked to both tonsillitis and healthy cohorts (Fig. 2). A limitation of using $16 \mathrm{~S}$ amplicon sequencing is the insufficient resolution in classifying species level differences [29, 30], which is compounded by microbial genera in oral communities encompassing multiple species and/or polyphyly in their phylogenetic classifications [3, 31]. Ideally, full-length $16 \mathrm{~S}$ sequences should be obtained to confirm exact species identities of any implicated microorganisms.

While the main aim of this study was to describe differences in oral community composition between patients with tonsillitis and healthy individuals, we also observed secondary differences in community composition linked to smoking in both healthy and tonsillitis cohorts. Firstly, we wish to point out that smokers and former smokers were underrepresented in numbers compared to non-smokers (29 smokers, 19 former smokers and 160 non-smokers in total). Nevertheless, we identified associations of multiple Fusobacterium ESVs with smokers and Neisseria with non-smokers in both the tonsillitis (Fig. 3) and healthy cohorts (Additional file 1: Figure S3) consistent with other studies comparing oral community composition between smokers and non-smokers. Previous oral community surveys have consistently reported reductions of Neisseria detected in mucosal surfaces [32], oropharynx [33] and oral rinses [34-36] of smokers compared to nonsmokers. Conversely, taxa such as Veillonella, Actinomyces and Fusobacterium have been found to be increased in the oral cavity of smokers compared to non-smokers, although contrasting results exist [33-36] possibly due to differences in biogeography of samples and technical limitations of the $16 \mathrm{~S}$ gene in delineating microbial species (as mentioned above). In addition, we identified association of a Bergeyella and a member of candidate phylum SR1 with non-smokers, which was also found in non-smokers from a New York City cohort [37]. One notion of how smoking influences the oral microbial community composition is that cigarette smoke creates anaerobic conditions favouring anaerobic microorganisms such as Veillonella, Fusobacterium and Actinomyces, and suppresses aerobes such as Neisseria [32, 35, 37]. This inference is consistent with our data showing associations of other anaerobic or microaerophilic taxa such as Fusobacterium, Leptotrichia, Porphyromonas, Lactobacillus and Treponema in smokers from the tonsillitis and healthy cohorts, although whether they are linked to a predisposition to disease in smokers remains an open question. Most notably, Fusobacterium,
Leptotrichia and Rothia in the oral cavity have been associated with squamous cell carcinoma [38] and oral leukoplakia [39], and thus their association with smokers suggests that smoking habits may exacerbate oral cancers through the enrichment and/or activity of these microorganisms. However, as mentioned earlier, $16 \mathrm{~S}$ amplicon sequencing does not provide sufficient resolution in identifying species level associations. Additional studies are required to validate whether the microbial taxa implicated here indeed have oncogenic potential, and play a role in the link between smoking, microorganisms and oral cancer [40].

Although it is widely accepted that antibiotics exert a strong influence on the composition of human microbiomes, we detected no statistical difference in the oral rinse communities of patients provided with systemic antibiotics compared to those without. We wish to clarify that we likely saw no measurable effects of antibiotics consumption on the oral microbiome in this cohort because the use of antibiotics among subjects was highly variable (15 of 43 subjects received antibiotics; several classes of antibiotics) and not controlled as these were prescribed by general practitioners prior to subjects' admission into the hospital. Therefore, findings relating to the consumption of antibiotics should be interpreted with caution.

\section{Conclusion}

In conclusion, we found that the oral rinse microbial community composition is altered during tonsillitis compared to healthy individuals. Specifically, there was an enrichment of Prevotella taxa in the tonsillitis cohort, raising the possibility of using targeted antimicrobial interventions to treat acute tonsillitis by eradicating Prevotella species. We also identified a link with smoking habits independent of tonsillitis; several fusobacterial taxa were enriched in smokers compared to nonsmokers, further supporting a microbial link between smoking and oral cancers. The nature of these fusobacterial taxa as well as their associations with smoking and oral cancers should be investigated in more detail.

\section{Supplementary information}

Supplementary information accompanies this paper at https://doi.org/10. 1186/s12903-019-0956-5.

Additional file 1: Figure S1. Alpha diversity of oral rinse microbial communities in healthy individuals and patients with tonsillitis. Figure S2. Top 10 genera discriminating oral rinse microbial communities between tonsillitis and non-tonsillitis cohorts. Figure S3. Top exact sequence variants associated with smokers and former smokers vs nonsmokers in the non-tonsillitis cohort ranked according to effect size.

Table S1. PERMANOVA of oral microbial community composition in tonsillitis cohort. Table S2. PERMANOVA of oral microbial community composition in non-tonsillitis cohort

Additional file 2. Demographics questionnaire 


\section{Abbreviations}

DNA: Deoxyribonucleic acid; dNTP: deoxynucleoside triphosphate; ESV: Exact sequence variants; LEfSe: Linear discriminant analysis effect size; PCA: Principal component analysis; PCoA: Principal coordinate analysis; PCR: Polymerase chain reaction; PERMANOVA: Permutational multivariate analysis of variance; rRNA: Ribosomal ribonucleic acid

\section{Acknowledgements}

We wish to thank sample donors for participating in this study.

\section{Authors' contributions}

YKY analyzed and interpreted the data, and wrote the manuscript. MHC contributed to study conception and design, recruited subjects and proofread the manuscript. ZC assisted with data acquisition and proofread the manuscript. EWHL contributed to study design and recruited subjects. PYW performed laboratory work including processing samples, DNA isolation and sequencing library prep. CMN contributed to study conception and design, recruited subjects and proofread the manuscript. PKSC contributed to study conception and design, recruited subjects and was a major contributor in writing the manuscript; $\mathrm{MH}$ contributed to study conception and design, recruited subjects and critically revised the manuscript. All authors read and approved the final manuscript.

\section{Funding}

This study was supported by a seed fund for gut microbiota research provided by the Faculty of Medicine, The Chinese University of Hong Kong. The funding body had no role in study design, data collection and analyses, decision to publish or preparation of the manuscript.

\section{Availability of data and materials}

Raw sequence data generated for this study are available in the Sequence Read Archive under BioProject accession PRJNA559766. Patient data are available from the corresponding author on reasonable request.

\section{Ethics approval and consent to participate}

This study has been approved by the Kowloon West Cluster Research Ethics Committee (reference number KW/EX-16-155[103-08]). Written informed consent was obtained from all participants prior to collecting oral rinse samples.

\section{Consent for publication}

Not applicable.

\section{Competing interests}

The authors declare that they have no competing interests.

\section{Author details}

${ }^{1}$ Centre for Gut Microbiota Research, Faculty of Medicine, The Chinese University of Hong Kong, Hong Kong SAR, China. ${ }^{2}$ Department of Microbiology, Faculty of Medicine, The Chinese University of Hong Kong, Hong Kong SAR, China. ${ }^{3}$ Li Ka Shing Institute of Health Sciences, Faculty of Medicine, The Chinese University of Hong Kong, Hong Kong SAR, China. ${ }^{4}$ Department of Otorhinolaryngology, Head and Neck Surgery, Yan Chai Hospital, Hong Kong SAR, China.

Received: 20 August 2019 Accepted: 11 November 2019 Published online: 05 December 2019

\section{References}

1. The NIH HMP Working Group, Peterson J, Garges S, Giovanni M, McInnes P, Wang L, Schloss JA, Bonazzi V, McEwen JE, Wetterstrand KA, et al. The NIH human microbiome project. Genome Res. 2009;19(12):2317-23.

2. Chen T, Yu W-H, Izard J, Baranova OV, Lakshmanan A, Dewhirst FE. The Human Oral Microbiome Database: a web accessible resource for investigating oral microbe taxonomic and genomic information. Database. 2010;2010:baq013.

3. Dewhirst FE, Chen T, Izard J, Paster BJ, Tanner ACR, Yu W-H, Lakshmanan A, Wade WG. The human Oral microbiome. J Bacteriol. 2010;192(19):5002-17.

4. Welch JLM, Rossetti BJ, Rieken CW, Dewhirst FE, Borisy GG. Biogeography of a human oral microbiome at the micron scale. Proc Natl Acad Sci U S A. 2016;113(6):E791-800.
5. Welch JLM, Dewhirst FE, Borisy GG. Biogeography of the Oral microbiome: the site-specialist hypothesis. Annu Rev Microbiol. 2019;73. https://doi.org/ 10.1146/annurev-micro-090817-062503.

6. Han YW, Wang X. Mobile microbiome: Oral Bacteria in extra-oral infections and inflammation. J Dent Res. 2013;92(6):485-91.

7. Wade WG. The oral microbiome in health and disease. Pharmacol Res. 2013; 69(1):137-43.

8. Brook I, Yocum P, Friedman EM. Aerobic and anaerobic Bacteria in tonsils of children with recurrent tonsillitis. Ann Otol Rhinol Laryngol. 1981;90(3):261-3.

9. Brook I. The role of anaerobic bacteria in tonsillitis. Int J Pediatr Otorhinolaryngol. 2005;69(1):9-19.

10. Nord CE. The role of anaerobic Bacteria in recurrent episodes of sinusitis and tonsillitis. Clin Infect Dis. 1995;20(6):1512-24

11. Rosenbaum J, Usyk M, Chen Z, Zolnik CP, Jones HE, Waldron L, Dowd JB, Thorpe LE, Burk RD. Evaluation of Oral cavity DNA extraction methods on bacterial and fungal microbiota. Sci Rep. 2019;9(1):1531.

12. Jensen A, Fagö-Olsen $H$, Sørensen $C H$, Kilian M. Molecular mapping to species level of the Tonsillar crypt microbiota associated with health and recurrent tonsillitis. PLoS One. 2013;8(2):e56418.

13. Lim Y, Fukuma N, Totsika M, Kenny L, Morrison M, Punyadeera C. The performance of an Oral microbiome biomarker panel in predicting Oral cavity and Oropharyngeal cancers. Front Cell Infect Microbiol. 2018:8:267.

14. Lim Y, Totsika M, Morrison M, Punyadeera C. The saliva microbiome profiles are minimally affected by collection method or DNA extraction protocols. Sci Rep. 2017;7(1):8523.

15. Wang L, Yin G, Guo Y, Zhao Y, Zhao M, Lai Y, Sui P, Shi T, Guo W, Huang Z. Variations in Oral microbiota composition are associated with a risk of throat Cancer. Front Cell Infect Microbiol. 2019;9:205.

16. Yeoh YK, Chen Z, Hui M, Wong MCS, Ho WCS, Chin ML, Ng SC, Chan FKL, Chan PKS. Impact of inter- and intra-individual variation, sample storage and sampling fraction on human stool microbial community profiles. PeerJ. 2019;7:e6172.

17. Bolyen E, Rideout JR, Dillon MR, Bokulich NA, Abnet CC, Al-Ghalith GA, et al. Reproducible, interactive, scalable and extensible microbiome data science using QIIME 2. Nat Biotechnol. 2019;37(8):852-7.

18. Quast C, Pruesse E, Yilmaz P, Gerken J, Schweer T, Yarza P, Peplies J, Glöckner FO. The SILVA ribosomal RNA gene database project: improved data processing and web-based tools. Nucleic Acids Res. 2013:41(D1):D590-6.

19. Lê Cao K-A, Costello M-E, Lakis VA, Bartolo F, Chua X-Y, Brazeilles R, Rondeau P. MixMC: a multivariate statistical framework to gain insight into microbial communities. PLoS One. 2016;11(8):e0160169.

20. Segata N, Izard J, Waldron L, Gevers D, Miropolsky L, Garrett WS, Huttenhower C. Metagenomic biomarker discovery and explanation. Genome Biol. 2011;12(6):R60.

21. La Rosa PS, Brooks JP, Deych E, Boone EL, Edwards DJ, Wang Q, Sodergren E, Weinstock G, Shannon WD. Hypothesis testing and power calculations for taxonomic-based human microbiome data. PLoS One. 2012;7(12):e52078.

22. Rajasuo A, Jousimies-Somer H, Savolainen S, Leppänen J, Murtomaa H, Meurman JH. Bacteriologic findings in tonsillitis and Pericoronitis. Clin Infect Dis. 1996;23(1):51-60.

23. Lundmark A, Hu Y, Huss M, Johannsen G, Andersson AF, Yucel-Lindberg T. Identification of salivary microbiota and its association with host inflammatory mediators in periodontitis. Front Cell Infect Microbiol. 2019:9:216.

24. Schwarzberg K, Le R, Bharti B, Lindsay S, Casaburi G, Salvatore F, Saber MH, Alonaizan F, Slots J, Gottlieb RA, et al. The personal human Oral microbiome obscures the effects of treatment on periodontal disease. PLoS One. 2014; 9(1):e86708.

25. Liu B, Faller LL, Klitgord N, Mazumdar V, Ghodsi M, Sommer DD, Gibbons TR, Treangen TJ, Chang YC, Li S, et al. Deep sequencing of the Oral microbiome reveals signatures of periodontal disease. PLoS One. 2012;7(6):e37919.

26. Gomes BPFA, Jacinto RC, Pinheiro ET, Sousa ELR, Zaia AA, Ferraz CCR, SouzaFilho FJ. Porphyromonas gingivalis, Porphyromonas endodontalis, Prevotella intermedia and Prevotella nigrescens in endodontic lesions detected by culture and by PCR. Oral Microbiol Immunol. 2005:20(4):211-5.

27. Takahashi N, Yamada T. Pathways for amino acid metabolism by Prevotella intermedia and Prevotella nigrescens. Oral Microbiol Immunol. 2000;15(2):96-102.

28. Takahashi N, Sato T. Dipeptide utilization by the periodontal pathogens Porphyromonas gingivalis, Prevotella intermedia, Prevotella nigrescens and Fusobacterium nucleatum. Oral Microbiol Immunol. 2002;17(1):50-4. 
29. Golob JL, Margolis E, Hoffman NG, Fredricks DN. Evaluating the accuracy of amplicon-based microbiome computational pipelines on simulated human gut microbial communities. BMC Bioinformatics. 2017;18(1):283.

30. Almeida A, Mitchell AL, Tarkowska A, Finn RD. Benchmarking taxonomic assignments based on $16 \mathrm{~S}$ rRNA gene profiling of the microbiota from commonly sampled environments. Gigascience. 2018;7(5):giy054.

31. Abranches J, Zeng L, Kajfasz JK, Palmer SR, Chakraborty B, Wen ZT, Richards VP, Brady LJ, Lemos JA. Biology of Oral Streptococci. Microbiol Spectr. 2018;6(5): GPP3-0042-2018. https:/doi.org/10.1128/microbiolspec.GPP3-0042-2018

32. Colman G, Beighton D, Chalk AJ, Wake S. Cigarette smoking and the microbial flora of the mouth. Aust Dent J. 1976;21(2):111-8,

33. Charlson ES, Chen J, Custers-Allen R, Bittinger K, Li H, Sinha R, Hwang J, Bushman FD, Collman RG. Disordered microbial communities in the upper respiratory tract of cigarette smokers. PLoS One. 2010;5(12):e15216.

34. Morris A, Beck JM, Schloss PD, Campbell TB, Crothers K, Curtis JL, Flores SC, Fontenot AP, Ghedin E, Huang L, et al. Comparison of the respiratory microbiome in healthy nonsmokers and smokers. Am J Respir Crit Care Med. 2013;187(10):1067-75

35. Wu J, Peters BA, Dominianni C, Zhang Y, Pei Z, Yang L, Ma Y, Purdue MP, Jacobs EJ, Gapstur SM, et al. Cigarette smoking and the oral microbiome in a large study of American adults. ISME J. 2016;10:2435-46.

36. Börnigen D, Ren B, Pickard R, Li J, Ozer E, Hartmann EM, Xiao W, Tickle T, Rider J, Gevers D, et al. Alterations in oral bacterial communities are associated with risk factors for oral and oropharyngeal cancer. Sci Rep. 2017; 7(1):17686.

37. Beghini F, Renson A, Zolnik CP, Geistlinger L, Usyk M, Moody TU, Thorpe L, Dowd JB, Burk R, Nicola S, et al. Tobacco exposure associated with oral microbiota oxygen utilization in the New York City Health and Nutrition Examination Study. Ann Epidemiol. 2019;34:18-25.e3.

38. Yang CY, Yeh YM, Yu HY, Chin CY, Hsu CW, Liu H, Huang PJ, Hu SN, Liao CT, Chang KP, et al. Oral microbiota community dynamics associated with Oral squamous cell carcinoma staging. Front Microbiol. 2018;9:862.

39. Amer A, Galvin S, Healy CM, Moran GP. The microbiome of potentially malignant Oral leukoplakia exhibits enrichment for Fusobacterium, Leptotrichia, Campylobacter, and Rothia species. Front Microbiol. 2017;8:2391.

40. Hooper SJ, Wilson MJ, Crean SJ. Exploring the link between microorganisms and oral cancer: a systematic review of the literature. Head Neck. 2009;31(9): 1228-39.

\section{Publisher's Note}

Springer Nature remains neutral with regard to jurisdictional claims in published maps and institutional affiliations.

Ready to submit your research? Choose BMC and benefit from:

- fast, convenient online submission

- thorough peer review by experienced researchers in your field

- rapid publication on acceptance

- support for research data, including large and complex data types

- gold Open Access which fosters wider collaboration and increased citations

- maximum visibility for your research: over $100 \mathrm{M}$ website views per year

At $\mathrm{BMC}$, research is always in progress.

Learn more biomedcentral.com/submissions 